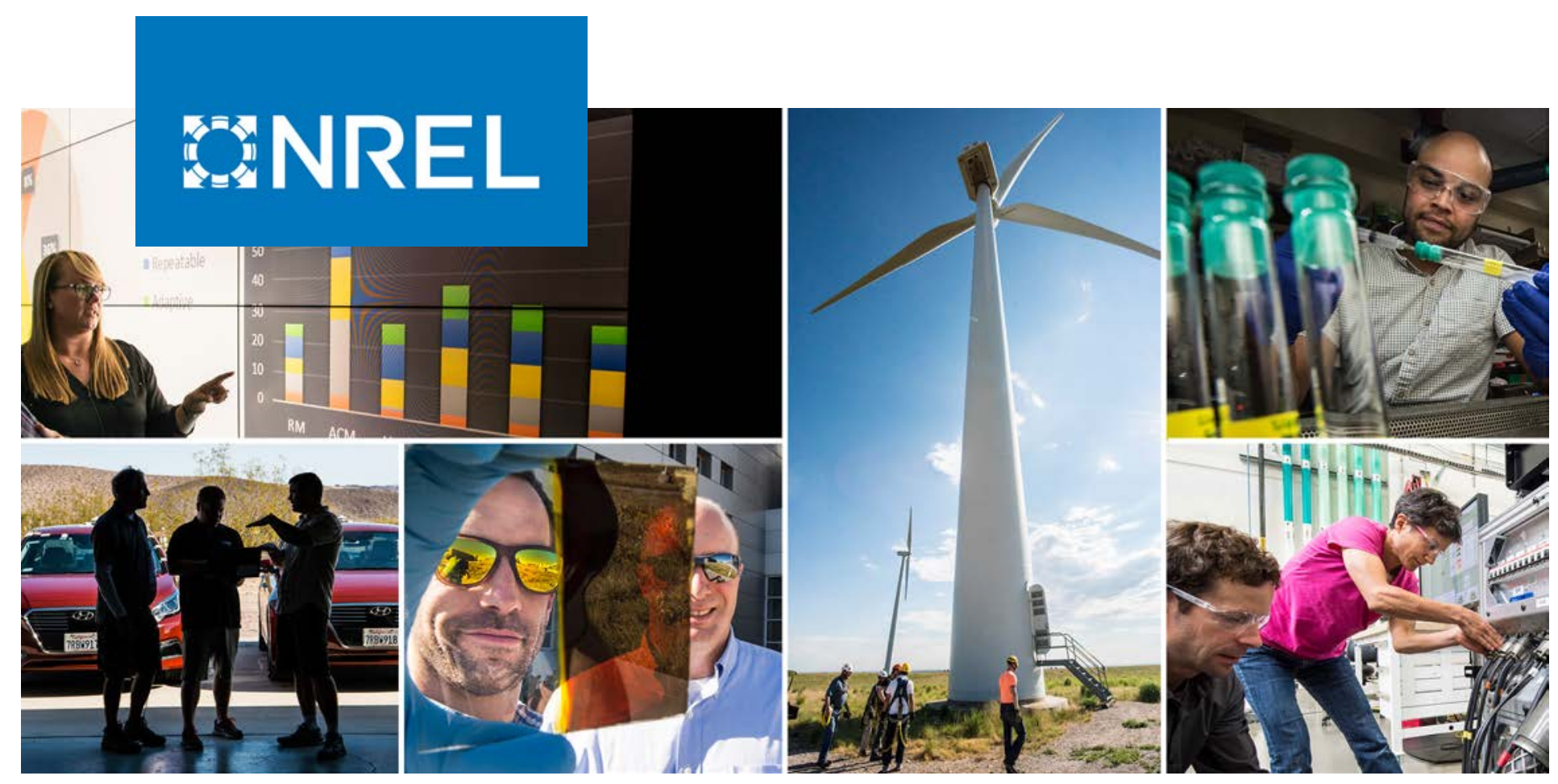

\title{
The Role of Interfacial Thermal Resistance in Li-Ion Battery Thermal Management
}

\section{Preprint}

Chuanbo Yang and Lei Cao

National Renewable Energy Laboratory

Presented at ASME 2019 International Technical Conference and Exhibition on Packaging and Integration of Electronic and Photonic Microsystems (IPACK2019) Anaheim, California

October 7-9, 2019

NREL is a national laboratory of the U.S. Department of Energy Office of Energy Efficiency \& Renewable Energy

Operated by the Alliance for Sustainable Energy, LLC

This report is available at no cost from the National Renewable Energy Laboratory (NREL) at www.nrel.gov/publications.

\section{Conference Paper}

NREL/CP-5400-73955

February 2020 


\section{GNREL}

\section{The Role of Interfacial Thermal}

Resistance in Li-Ion Battery Thermal

Management

\section{Preprint}

Chuanbo Yang and Lei Cao

National Renewable Energy Laboratory

\section{Suggested Citation}

Yang, Chuanbo, and Lei Cao. 2020. The Role of Interfacial Thermal Resistance in Li-Ion Battery Thermal Management: Preprint. Golden, CO: National Renewable Energy Laboratory. NREL/CP-5400-73955. https://www.nrel.gov/docs/fy20osti/73955.pdf.

NREL is a national laboratory of the U.S. Department of Energy Office of Energy Efficiency \& Renewable Energy Operated by the Alliance for Sustainable Energy, LLC

This report is available at no cost from the National Renewable Energy Laboratory (NREL) at www.nrel.gov/publications.

Contract No. DE-AC36-08GO28308
Conference Paper NREL/CP-5400-73955

February 2020

National Renewable Energy Laboratory 15013 Denver West Parkway Golden, CO 80401 303-275-3000 • www.nrel.gov 


\section{NOTICE}

This work was authored by the National Renewable Energy Laboratory (NREL), operated by Alliance for Sustainable Energy, LLC, for the U.S. Department of Energy (DOE) under Contract No. DE-AC36-08GO28308. Funding provided by U.S. Department of Energy Office of Energy Efficiency and Renewable Energy Vehicle Technologies Office. The views expressed in the article do not necessarily represent the views of the DOE or the U.S. Government. The U.S. Government retains and the publisher, by accepting the article for publication, acknowledges that the U.S. Government retains a nonexclusive, paid-up, irrevocable, worldwide license to publish or reproduce the published form of this work, or allow others to do so, for U.S. Government purposes.

This report is available at no cost from the National Renewable Energy Laboratory (NREL) at www.nrel.gov/publications.

U.S. Department of Energy (DOE) reports produced after 1991 and a growing number of pre-1991 documents are available free via www.OSTI.gov.

Cover Photos by Dennis Schroeder: (clockwise, left to right) NREL 51934, NREL 45897, NREL 42160, NREL 45891, NREL 48097, NREL 46526.

NREL prints on paper that contains recycled content. 


\title{
The Role of Interfacial Thermal Resistance in Li-Ion Battery Thermal Management
} Chuanbo Yang, ${ }^{\mathrm{a},}$, Lei Cao ${ }^{\mathrm{a}}$

${ }^{a}$ National Renewable Energy Laboratory, 15013 Denver West Parkway, Golden, CO 80401, USA *Corresponding author: Chuanbo Yang, National Renewable Energy Laboratory

\begin{abstract}
Temperature critically affects the performance, life and safety of lithium-ion batteries. Therefore, it is essential to understand heat generation and dissipation within individual battery cells and battery packs to plan a proper thermal management strategy. One of the key challenges is that interfacial heat transfer of a battery unit is difficult to quantify. The steady-state absolute method and the transient laser-flash-diffusivity method were employed to measure heat conductivities of battery layer stacks and individual battery layer separately. Results show flash diffusivity method gives higher thermal conductivity at both cross-plane and in-plane directions. The difference is primarily caused by interfacial thermal resistance so that it can be estimated by steady-state and transient measurements.

To investigate the effects of interfacial thermal transport beyond individual cell level, a multiphysics battery model is used. The model is built upon a multi-scale multi-domain modeling framework for battery packs that accounts for the interplay across multiple physical phenomena. Benefits of a battery module using thermal management materials are quantified through numerical experiments. During a thermal runaway event, it is found interfacial thermal resistance can mitigate thermal runaway in a battery module by significantly reducing heat transfer between cells.
\end{abstract}

\section{Keywords:}

Li-ion battery, thermal management, interfacial thermal resistance, multiphysics modeling

$\begin{array}{ll}\text { Nomenclature } \\ \mathrm{T} & \text { Temperature } \\ \mathrm{k} & \text { Thermal conductivity } \\ \alpha & \text { Thermal diffusivity } \\ \rho & \text { Density } \\ \mathrm{C}_{\mathrm{p}} & \text { Heat capacity } \\ l_{\mathrm{i}} & \text { Thickness }\end{array}$

\section{Introduction}

Performance and life of li-ion batteries are strongly influenced by absolute temperature and uniformity of temperature control [1]. Under abusive conditions such as internal short circuits, heat generated may raise battery temperature to trigger exothermic chemical reactions of battery components and lead to thermal runaway when heat is not dissipated efficiently. Battery thermal management systems (BTMS) are essential to prevent the occurrence of thermal issues in battery energy storage systems. The goal becomes even more important for battery thermal management of high energy density cells under extreme fast charging. State-of-the-art BTMS technologies have been discussed by Wang and Kim et.al [2,3]. A promising method for large-format pouch cells is fin-cooling in which a liquid cold plate underneath the batteries is thermally connected to aluminum fins between battery cells. 
Based on USABC requirements on li-ion battery thermal management [4], a battery pack should work at a temperature range from $-30{ }^{\circ} \mathrm{C}$ to $52^{\circ} \mathrm{C}$ and pack temperature uniformity shall be less than $3{ }^{\circ} \mathrm{C}$. To maintain battery pack temperature and minimize temperature gradients, Li-ion battery thermal conductivity and interfacial thermal resistances are critical. Because of the structural properties of multi-layer stacked porous electrode, Li-ion battery has a much larger inplane thermal conductivity than that in the cross-plane. Temperature gradient is usually more significant along its thickness direction. To develop effective BTMS, it is important to understand the effect of anisotropic heat transfer behaviors.

Modeling has been recognized to be robust for design of BTMSs, which is a low-cost approach to evaluate design tradeoffs and quantify thermal and electrochemical inhomogeneity that is too expensive to determine with experiments. Funded by the U.S. Department of Energy's ComputerAided Engineering for Electric Drive Vehicle Batteries (CAEBAT) program, a multi-scale multidomain (MSMD) modeling framework [5] has been in implemented in ANSYS Fluent commercial software [6]. The model considers battery geometry into three coupled domains, including particle-, electrode- and cell domain. Modeling domain separation for the physicochemical process interplay is carried out where the characteristic time or length scale is segregated. The modeling framework is expandable, modular, and flexible that connects battery Multiphysics in a computationally efficient manner.

\section{Methodology}

A combined experimental and modeling approach is employed to study the effects of thermal resistances in a battery module. Experiments were conducted to measure anisotropic heat conductivities of batteries. Multiphysics modeling is used to quantify its impact on the performance of BTMS.

\subsection{Thermal Conductivity Measurement}

It is difficult to directly measure thermal conductivity of Li-ion full cells. Without opening the cells, packaging material itself and the contact between packaging and electrodes decrease validity of results for electrodes. Whereas wet electrodes from opened cells suffer from evaporation of organic solvents and instability with moisture and air under measuring conditions. Therefore, a more practical way to determine thermal conductivities is through a lumped-component model. Thermal conductivities of stacked or individual dry layers are measured first while maintained at the same density of what's in the cell. Effective thermal conductivities of soaked porous layers can be calculated as a weighted mean of the conductivity corresponding to a parallel/series arrangement of the dry and wet phases. Ultimately, the overall effective thermal conductivity of a Li-ion battery is the weighted mean of each soaked layer's effective thermal conductivities, where the weight is the cross-sectional area of each layer. 

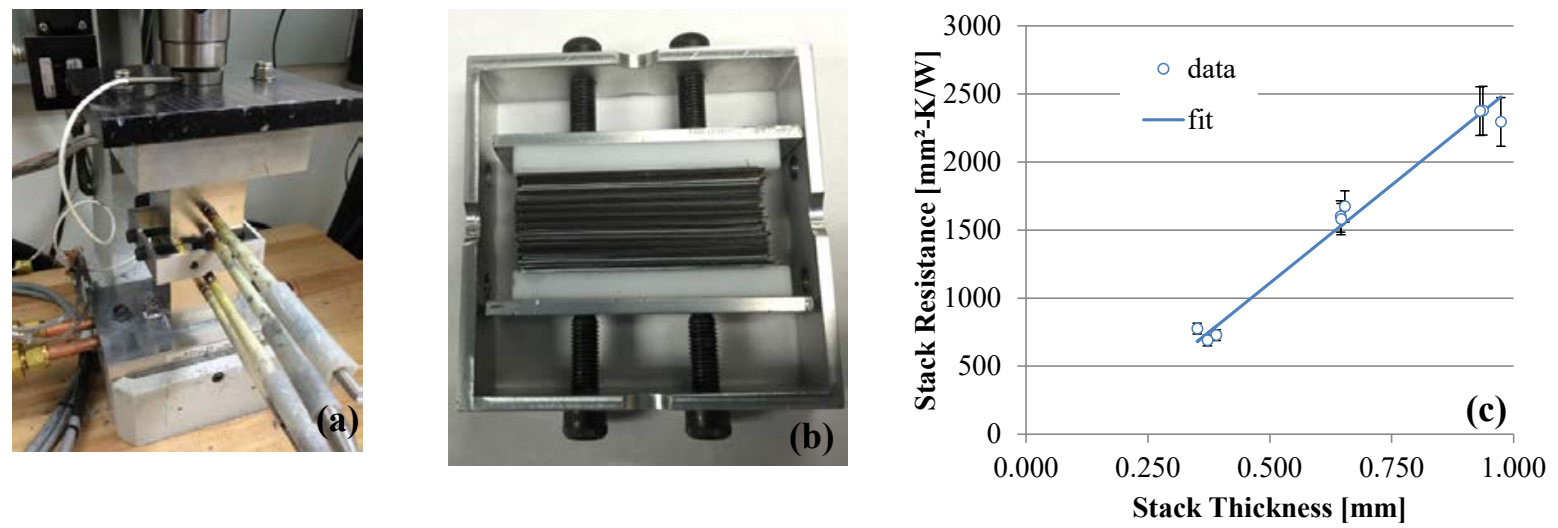

Figure 1: (a) Photo of experiment setup for the steady-state approach; (b) Photo of battery layer stacking for in-plane thermal conductivity test; (c) A sample of linear fitting of cross-plane thermal conductivity

Herein, two approaches were applied to measure thermal conductivities of cell components, including steady-state and transient approaches. Figurel (a) shows the setup for steady-state approach. The test apparatus was built according to ASTM Standard D5470-12. Battery electrode laminates are placed between two copper metering blocks, with layers in parallel with metering block surface or perpendicular to it, as shown in Figure 2 (b). Sample thermal resistance is measured when staple temperatures at hot and cold metering block surface are reached while applying a constant current to the heater. Total thermal resistances are then plot against stack thickness when varying numbers of stacked layers, shown in Figure 1 (c). Apparent thermal conductivity is the reciprocal of the slope of the fitted stack resistance at various lamination thickness. For multi-layer testing samples, this method provides overall thermal resistance of layer samples including contact resistance between layers.
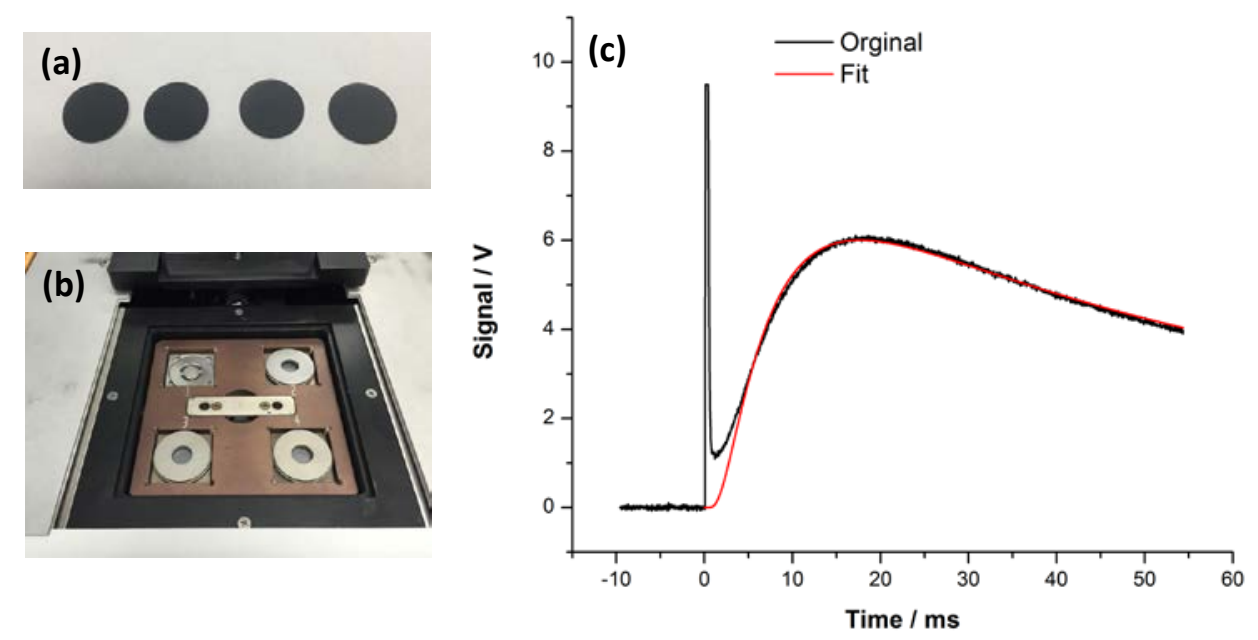

Figure 2: Testing procedures for the laser flash technique

On the other hand, the transient approach estimates single-layer sample thermal conductivity using instantly measured thermal diffusivity $\alpha$, effective density $\rho$ and effective heat capacity $\mathrm{Cp}$ described in Equation (1). A Nanoflash LFA 447 instrumentation from Netzsch is used to measure thermal diffusivity of individual battery layers through laser flash technique. Figure 2 (a) shows round samples used for holders in Figure 2 (b) of such a machine. When a pulse of xenon flash is shot at lower surface of sample, the temperature rise of the other side is measured as a function of 
time using an infrared detector. Thermal diffusivity is calculated through data fitting excluding the initial spike directly caused by xenon flash, as illustrated in Figure 2 (c). Compared against the steady-state method, the transient method using laser flash is for individual electrode layer and requires other thermal properties of testing samples, including bulk density and specific heat capacity.

$$
k(T)=\alpha(T) \rho(T) C_{p}(T)
$$

\subsection{Multiphysics Modeling}

Compared against interfacial thermal resistance within a battery cell, interfacial thermal resistance between battery cells and other battery pack accessories are even more challenging to measure, especially in a real large-size battery pack. To address its effects on thermal performance, a modeling approach is employed in this study. The integrated multiphysics model is built on the MSMD modeling framework [5] to investigate its effects at normal operating condition and under thermal abuse condition. A state variable model (SVM), a reduced order representation of 1D electrochemical model, is used to simulate battery electrochemical-thermal behaviors [7]. Additionally, an abuse reaction kinetics model [8] is included to estimate heat released by decomposition reactions of battery components at elevated temperatures. Details of the integrated modeling approach can be found at reference [9].
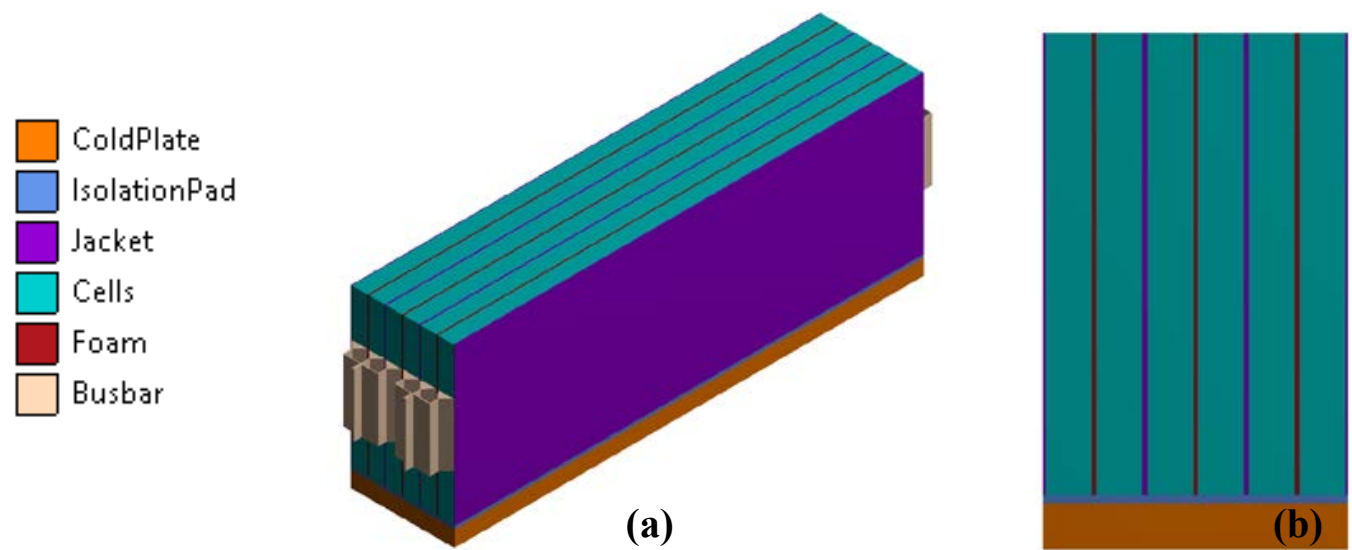

Figure 3: 3D geometry of the 3P2S module ((a) isometric view; (b) front view without busbar) 


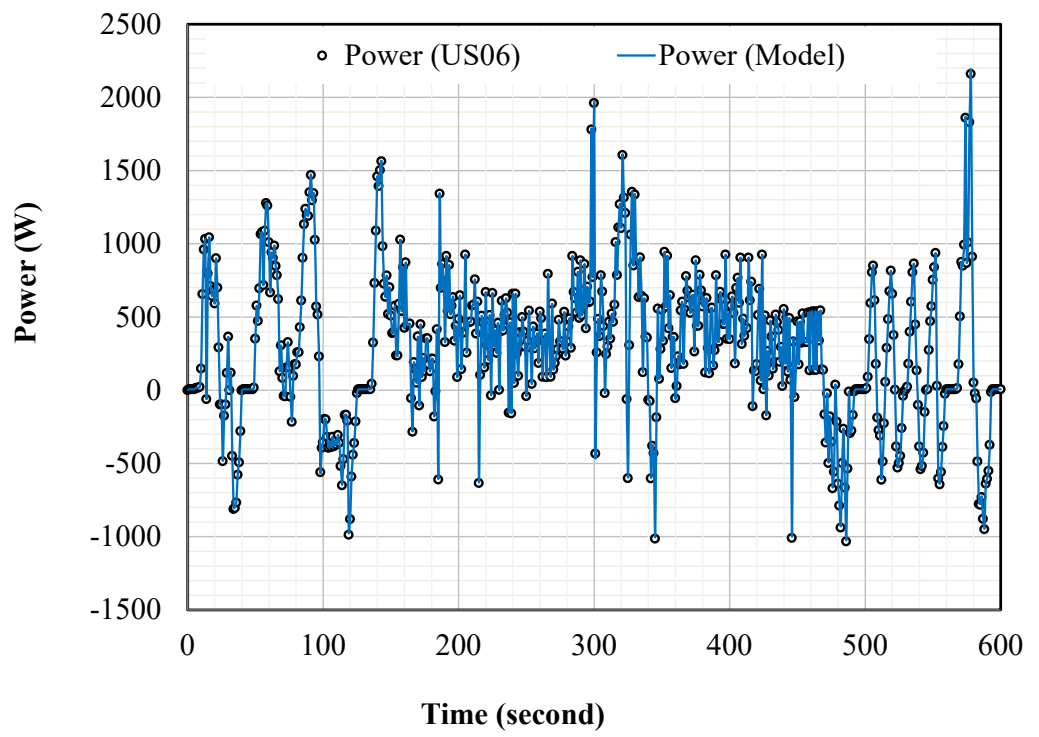

Figure 4: Power demands in EPA US06 drive cycle

Figure 3 shows the battery module for multiphysics modeling. Its electrical configuration has each of three cells connected first in parallel and then two parallel strings connected in series (3P2S). It is a basic repeating unit in term of electrical and thermal configuration in a large battery pack. The module temperate is controlled through a fin cooling technique, including cooling fins and a cold plate and a thermal gap pad between them. Thermally, every two battery cells are separated by a cooling fin, and there is foam between these two cells.

Table 1.3D simulation conditions

\begin{tabular}{|l|l|}
\hline Condition & Values \\
\hline Configuration & $3 \mathrm{P} 2 \mathrm{~S}$ \\
\hline Initial SOC & $100 \%$ \\
\hline Module loads & $\begin{array}{l}\text { US06 (normal operating) and open circuit } \\
\text { condition (abuse condition) }\end{array}$ \\
\hline Initial temperature & $23^{\circ} \mathrm{C}$ \\
\hline Boundary conditions & $\begin{array}{l}2{ }^{\circ} \mathrm{C} \text { at cold plate bottom surface and } \\
\text { adiabatic conditions elsewhere }\end{array}$ \\
\hline TCR, foams and cells & $10.0 \mathrm{~m}^{2}{ }^{\circ} \mathrm{C} \mathrm{kW}^{-1}$ \\
\hline TCR, case 1 & $0.10 \mathrm{~m}^{2} \mathrm{C} \mathrm{kW}^{-1}$ \\
\hline TCR, case 2 & $1.0 \mathrm{~m}^{2}{ }^{\circ} \mathrm{C} \mathrm{kW}^{-1}$ \\
\hline TCR, case 3 & $10.0 \mathrm{~m}^{2} \mathrm{C} \mathrm{kW}^{-1}$ \\
\hline
\end{tabular}

The BTMS performance is simulated under both nominal operating and abusive conditions entailed in Table 1. Under nominal operating conditions, the module loads are power-type scaled down from a full battery pack to support an electrical vehicle for EPA US06 drive cycle. Details of this cycle are shown in Figure 4. Under abusive conditions, the module is at open circuit condition. It is assumed the internal short circuit occurred at the middle of cell 3 , which produces a local hot spot and trigger exothermic decomposition reactions. This local thermal runaway propagates to other places in cell 3 . Only the thermal aspect is considered in the simulation so that 
the SVM model is decoupled. The only modeling parameter is the thermal contact resistance between cells and aluminum fins, as shown in Table 1.

\section{Results and Discussion}

\subsection{Testing Results}

Steady-state method directly gives bulk thermal conductivities considering the contact resistance between battery component layers. The transient method measures the thermal conductivities between individual layers. Directly comparing results from these two approaches is not possible. Instead, the effective thermal conductivities of a dry unit cell are extracted from transient measurements using the equations below. ki and li are the thermal conductivity and thickness of component i, respectively.

$$
k_{\text {cross-plane }}=\frac{\sum l_{i}}{\sum \frac{l_{i}}{k_{i}}} \quad k_{\text {in-plane }}=\frac{\sum k_{i} l_{i}}{\sum l_{i}}
$$

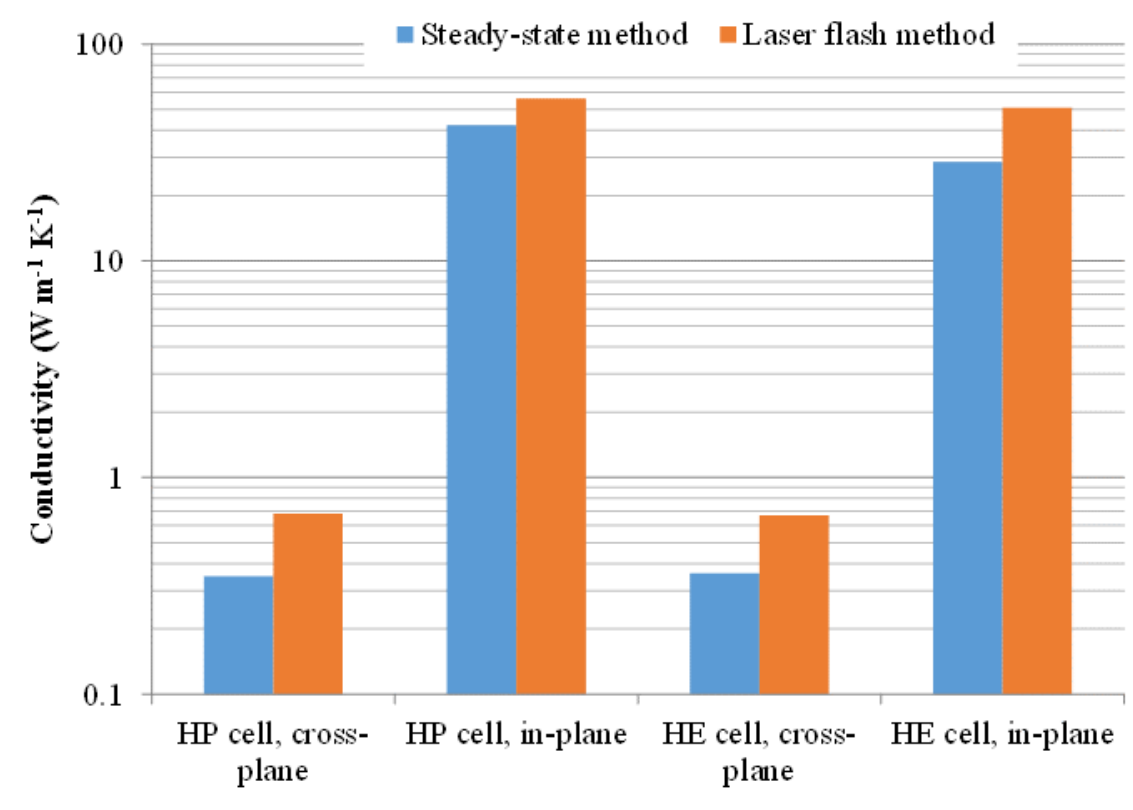

Figure 5: Heat conductivities for HP and HE cells

Figure 5 illustrates results for two cell designs: high power (HP) and high energy (HE) cells. In general, in-plane thermal conductivity is one to two orders of magnitude greater than the crossplane values, attributed to the contribution of current collector's higher-grade thermal conductivity than those of other components. In cross plane direction, high power and high energy cells have nearly identical thermal conductivity because the cell conductivity is dominated by porous polymer separator and electrode coating. For the in plane-direction, high power cell has a higher thermal conductivity because of the higher current collector contribution, which, made by copper and aluminum, has a higher thermal conductivity. High energy cells usually have a thicker electrode coating with a smaller contribution from current collectors. Notably, the conductivities from flash diffusivity method, with which no interfacial resistance is introduced in calculation, is higher than those from ASTM method. The cross-plane values are roughly twice of those from ASTM method and in-plane values show better agreement with around $50 \%$ difference. As 
suggested by ASTM Standard D5470-12, thermal interfacial resistance is included in the measurement and can be calculated through data-processing. Cross-plane measurement introduces interfacial resistance between the sample and metering block, and from within the sample. In the case of in-plane ASTM measurement, interfacial resistance is limited to only between sample and metering block. Moreover, calculation using flash diffusivity data is based on assumptions of ideal contact without interfacial resistance. Therefore, the values from flash diffusivity method are higher than those from ASTM measurement with a bigger difference in cross-plane direction.

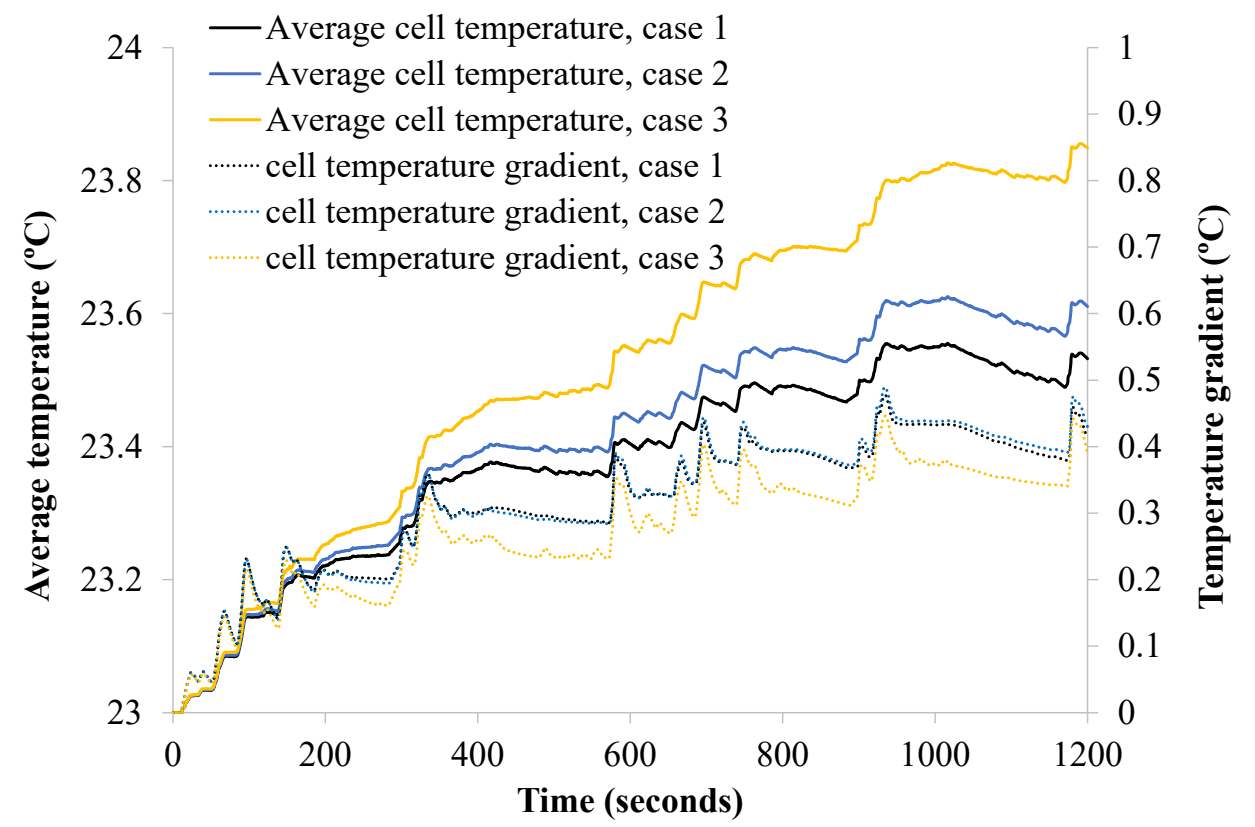

Figure 6: Temperature rise and gradients of the cells under nominal operating conditions

\subsection{Modeling Results}

Under the nominal operating conditions, the loading profiles to meet US06 drive power demands have been simulated for two cycles. Cell temperature and its gradients rises continuously until an equilibrium state is reached at the end, as shown in Figure 6. The temperature fluctuations are caused by the dynamics power pulses in the US06 profile. The average temperature rises of case1 to case 3 are $0.55,0.6$ and $0.85 \circ \mathrm{C}$ respectively near the end of the 2 nd cycle. The heat generated by the module are nearly identical due to the small temperature rise, which has a negligible effect on electrochemical modeling parameters. As such, the differences in temperature rise are mainly caused by different heat dissipation rate. Figure 7 shows the heat absorption rates of the cold plate, indicating higher thermal contact resistance (i.e. $10.0 \mathrm{~m}^{2}{ }^{\circ} \mathrm{C} \mathrm{kW}^{-1}$ ) in case 3 reduces the heat conduction between battery cells and the cold plate. However, simulation results show the temperature gradients among six cells are not significantly provoked, as plotted in Figure 6. 


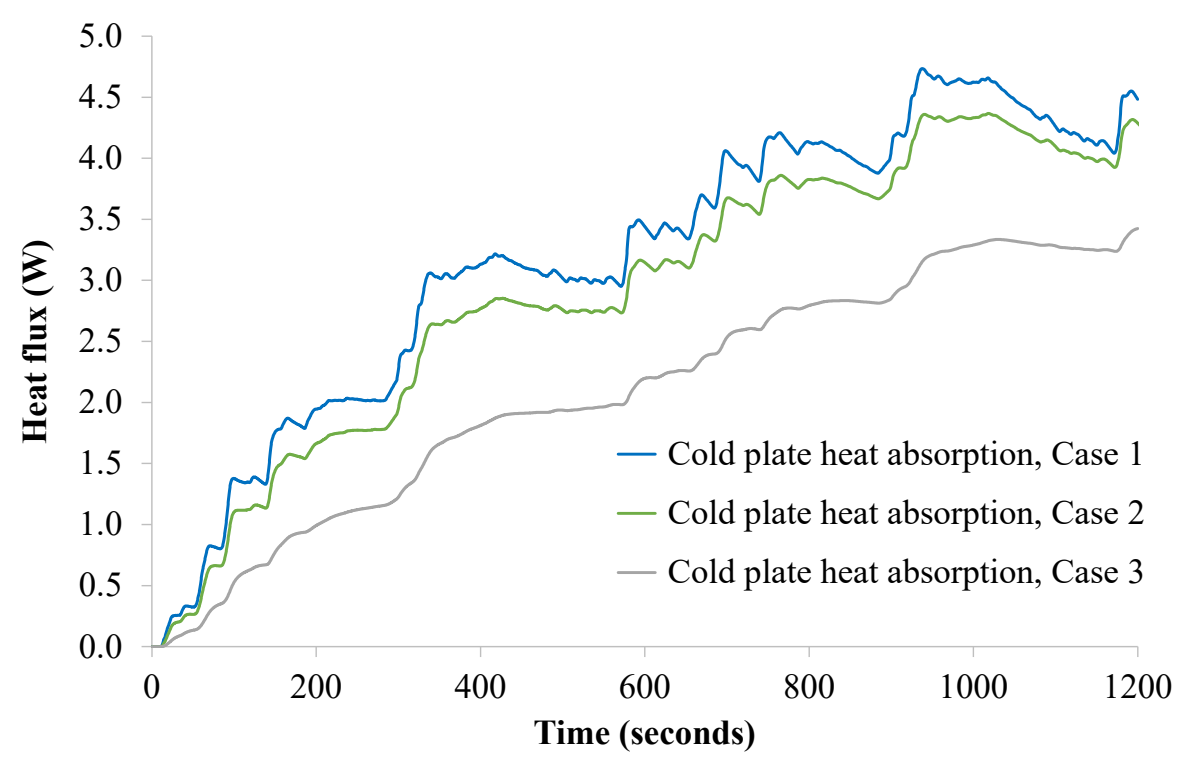

Figure 7: Heat absorption by the cold plate

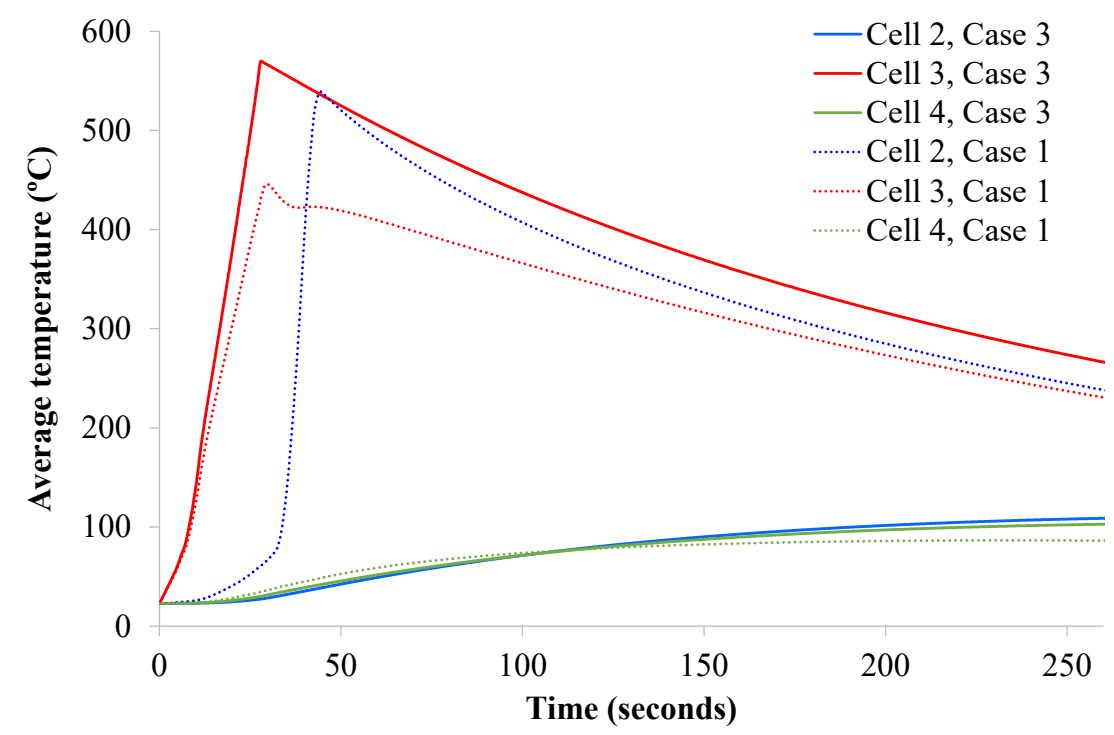

Figure 8: Cell average temperatures in the abuse event

Compared with nominal operating conditions, higher thermal contact resistances prevent adjacent cells from thermal shock produced by a failed cell. In Figure 8, the average temperature of the failure cell (cell 3) and its adjacent cells (cell 2 and 4) are plotted. For both case 1 and case 3, heat released by the failure cell is nearly identical because all the decomposition reactions are fully completed. With $10.0 \mathrm{~m}^{2}{ }^{\circ} \mathrm{C} \mathrm{kW}^{-1}$ of the thermal contact resistance, these adjacent cells remain safe. Their average temperatures are at about $100{ }^{\circ} \mathrm{C}$ after short circuit occurs at 250 seconds. When the thermal contact resistance decreased to be $0.1 \mathrm{~m}^{2}{ }^{\circ} \mathrm{C} \mathrm{kW}^{-1}$ in case 1 , thermal runaway propagates to cell 2 even the cell 3 has a relatively lower peak temperature due to fast heat dissipation to its surrounding components. This is because heat also transferred to cell 2 through the aluminum fin between them, which triggers the exothermic decomposition reactions in cell 2. 

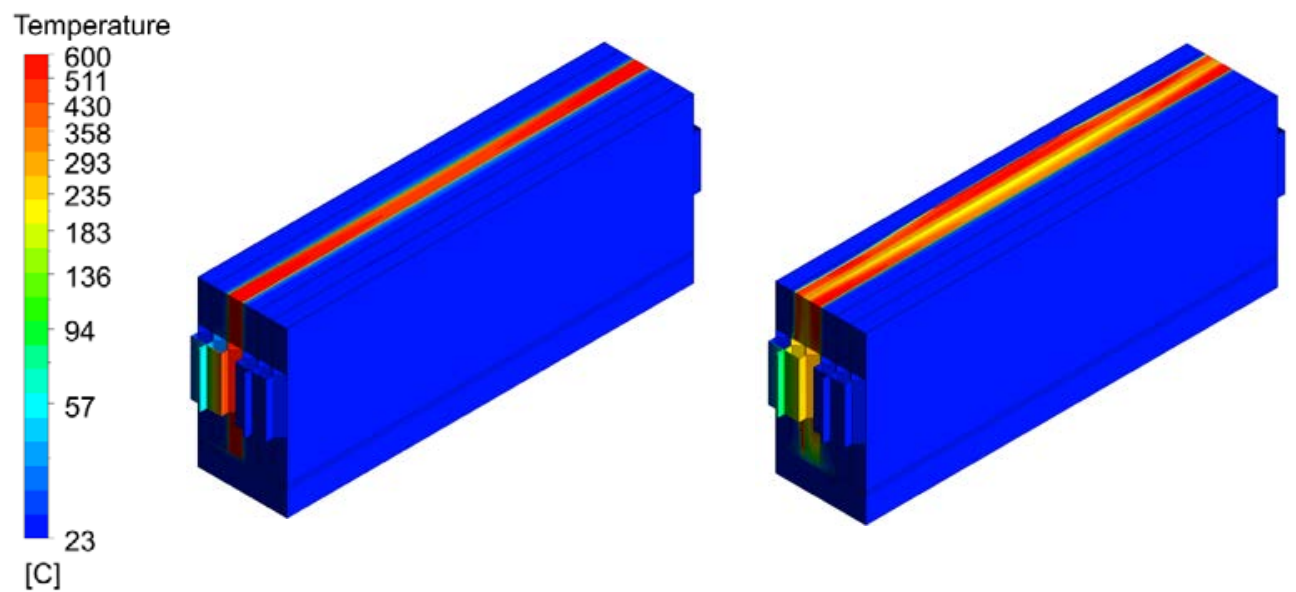

Figure 9: Temperature contours of the module at 40 seconds for case 1 (right) and 3 (left)

Figure 9 compares the temperature distributions of the module for case 1 and case 2 at 40 seconds after the occurrence of internal short circuit. As shown in Figure 9, higher thermal contact resistances can slow heat transfer between the failure cells and its adjacent components. Even for case 1, the temperature in cell 4 could be controlled because of the isolation foam between cell 3 and cell 4 . As such, the system has sufficient time to dissipate heat to the cold plate to maintain safety.

\section{Conclusions}

Anisotropic thermal conductivity of a battery cell can be determined by experiments using the steady-state and transient approaches. The measurement captures the difference caused by battery electrode design change. Thermal contact resistance negatively affects the performance of the BTMS at nominal operating conditions, resulting in temperature rise without obvious impact on battery internal temperature gradients. However, simulation results suggest thermal contact resistance may prevent cascading thermal runaway in a battery pack.

\section{Acknowledgements}

This work was authored in part by the National Renewable Energy Laboratory, operated by Alliance for Sustainable Energy, LLC, for the U.S. Department of Energy (DOE) under Contract No. DE-AC36-08GO28308. Funding provided by the U.S. Department of Energy Office of Energy Efficiency and Renewable Energy Vehicle Technologies Office Computer-Aided Engineering for Batteries (CAEBAT) Program, program manager Brian Cunningham. The views expressed in the article do not necessarily represent the views of the DOE or the U.S. Government. The U.S. Government retains and the publisher, by accepting the article for publication, acknowledges that the U.S. Government retains a nonexclusive, paid-up, irrevocable, worldwide license to publish or reproduce the published form of this work, or allow others to do so, for U.S. Government purposes. 


\section{References}

[1] Bandhauer, Todd M, Garimella, Srinivas and Fuller, Thomas F. "A Critical Review of Thermal Issues in Lithium-Ion Batteries." Journal of The Electrochemical Society Vol.158 No.3 (2011): pp. R1-R25.

[2] Wang, Qian, Jiang, Bin, Li, Bo and Yan, Yuying. "A critical review of thermal management models and solutions of lithium-ion batteries for the development of pure electric vehicles." Renewable and Sustainable Energy Reviews Vol.64 (2016): pp.106-128.

[3] Kim, Jaewan, Oh, Jinwoo and Lee, Hoseong. "Review on Battery Thermal Management System for Electric Vehicles.” Applied Thermal Engineering Vol.149 (2019): pp.192-212.

[4] USABC Li-Ion Battery Thermal Management System Requirements, 2018.

[5] Kim, Gi-Heon, Smith, Kandler, Lee, Kyu-Jin, Santhanagopalan, Shriram and Pesaran, Ahmad. "Multi-Domain Modeling of Lithium-Ion Batteries Encompassing Multi-Physics in Varied Length Scales.” Journal of The Electrochemical Society Vol.158 No.8 (2011): pp. A955-A969.

[6] ANSYS Fluent Advanced Add-On Modules, Release 16.0, January 2015, pp. 105-142.

[7] Smith, Kandler A, Rahn, Christopher D and Wang, Chao-Yang. "Control Oriented 1D Electrochemical Model of Lithium Ion Battery," Energy Conversion and Management Vol.48 No.9 (2007): pp. 2565-2578.

[8] Kim, Gi-Heon, Pesaran, Ahmad and Spotnitz, Robert. "A Three-Dimensional Thermal Abuse Model for Lithium-Ion Cells.” Journal of Power Sources Vol.170 No.2 (2007): pp. 476-489.

[9] Li, Qibo, Yang, Chuanbo, Santhanagopalan, Shriram, Smith, Kandler A, Lamb, Joshua, Steele, Leigh Anna, and Torres-Castro, Loraine. "Numerical investigation of thermal runaway mitigation through a passive thermal management system," Journal of Power Sources Vol.429 (2019): pp. 80-88. 